\title{
La séduction ou du sentiment de l'abandon (lecture de Camere separate de Pier Vittorio Tondelli
}

Flaviano Pisanelli

\section{(2) OpenEdition}

\section{Journals}

Édition électronique

URL : http://journals.openedition.org/cei/841

DOI : $10.4000 /$ cei.841

ISSN : 2260-779X

Éditeur

UGA Éditions/Université Grenoble Alpes

\section{Édition imprimée}

Date de publication : 15 septembre 2006

Pagination : 231-242

ISBN : 978-2-84310-086-4

ISSN : 1770-9571

\section{Référence électronique}

Flaviano Pisanelli, «La séduction ou du sentiment de l'abandon (lecture de Camere separate de Pier Vittorio Tondelli », Cahiers d'études italiennes [En ligne], 5 | 2006, mis en ligne le 15 mars 2008, consulté le 27 mars 2021. URL : http://journals.openedition.org/cei/841 ; DOI : https://doi.org/10.4000/cei.841 


\title{
LA SÉDUCTION OU DU SENTIMENT DE L’ABANDON
}

\author{
LECTURE DE CAMERE SEPARATE DE PIER VITTORIO TONDELLI
}

\author{
Flaviano Pisanelli \\ Université d'Avignon
}

\begin{abstract}
"I campi di battaglia, le morti quotidiane, la sopraffazione dell'altro avvengono in forma di "delitti sublimi» negli strati profondi della personalità e del comportamento. Non c'è, all'esterno, spargimento di sangue. Eppure i cadaveri sono fra di noi.»
\end{abstract}

Pier Vittorio Tondelli, Camere separate.

Publié en 1989, le roman Camere separate ${ }^{1}$ a été considéré comme l'œuvre de maturité de Pier Vittorio Tondelli. De nombreux critiques ont parlé de ce roman comme d'une forme d'intériorisation, de stratification, d'élaboration et de sublimation de toutes les vicissitudes de la jeunesse italienne des années quatre-vingt, qui constituent d'ailleurs la matière première de Altri libertini, de Pao pao et de Rimini. Mais dans son dernier roman, Tondelli prend ses distances avec le jargon et l'argot juvéniles, pour orchestrer des mouvements narratifs se fondant sur une mémoire et une intériorité qui se cachent et s'expriment par la force de la douleur. L'auteur devient ainsi le témoin d'une vie qui se confie à l'écriture et au sentiment de l'abandon, du séparé, de l'ambigu.

1. Tondelli P.V., Camere separate, Milan, Bompiani, 1989. Aujourd'hui, les œuvres complètes de l'auteur ont été publiées en deux volumes, sous la direction de F. Panzeri: Tondelli P.V., Opere. Romanzi, teatro, racconti, Milan, Bompiani, 2000 et Tondelli P.V., Opere. Cronache, saggi, conversazioni, Milan, Bompiani, 2001. Une traduction en langue française de Camere separate a été publiée en 1992: Tondelli P.V., Chambres séparées, trad. par N. Sels, Paris, Seuil, 1992. Dans notre étude, toute citation du texte fera référence à l'édition Bompiani de 1989, et sera suivie de l'indication de la page entre parenthèses. Pour un approfondissement ultérieur de l'œuvre de Tondelli, et en particulier de Camere separate, voir Minardi E., Pier Vittorio Tondelli, Fiesole, Cadmo, 2003, p. 88-96. 
À travers l'histoire d'un deuil - provoqué par la mort de l'amant et la déconstruction identitaire de soi-même qui en résulte -, Leo parcourt, requalifie, transforme, détruit et régénère son image en revivant le voyage de son existence.

Ce parcours, qui demande au protagoniste Leo de renoncer à son idéal et de comprendre pour de bon le sens de sa diversité (celle d'être simplement un homme parmi d'autres hommes, un écrivain, un homosexuel), s'accomplit grâce à une attitude de séduction, qui représente le point de départ et l'achèvement de son voyage dans le deuil, dans la désillusion, jusqu'à la prise de conscience d'être un «homme séparé», dans le sens de «différent».

Pour mieux comprendre la fonction et le sens de la séduction à l'intérieur de ce roman, il nous faut prendre en compte l'étymologie du mot "séduction", qui dérive du latin "seductio-onis». Cicéron utilise ce terme dans le sens de "mettre quelqu'un à l'écart»; Célius Firmin Lactance l'emploie dans le sens de "séparation». Ce substantif renvoie au verbe latin «seduco» qui, dans ses différentes acceptions, se rapporte à l'action de «diviser", "séparer", «distinguer», "se conduire, se porter ailleurs».

La notion de séduction s'impose dans le projet ultime du roman. Elle conduit, amène, ramène, trace et efface, unit les protagonistes et les sépare. La vie de l'un représente toujours la mort de l'autre: dans ce flux bipolaire entre la vie et la mort, Leo et Thomas expérimentent l'apprentissage d'un amour qui fuit l'absolu et s'achève par la mort de Thomas. Ce moment marquera le début de la régénération de Leo par la découverte d'une solitude qui sera perçue, enfin, comme une valeur et non pas comme une nécessité.

Camere separate n'est structuré ni en chapitres ni en épisodes. L'ouvrage se compose de trois mouvements qui mettent l'accent sur les notions de transformation, de métamorphose, sur le caractère initiatique du texte, dans une sorte de concordia discordabilis, la condition naturelle de l'identité humaine ${ }^{2}$. Dans ce parcours vers la régénération, tous les

2. À propos de la structure narrative de Camere separate, Tondelli, lors d'un entretien avec Fulvio Panzeri, dit: «Avec Camere separate je ne voulais pas créer une sorte de confession. Je devais trouver une forme: j’ai alors pensé à une forme musicale qui est beaucoup plus spontanée et authentique, car cette narration est comme un chant. C'est le chant d'une personne seule qui réfléchit, qui reprend tout son passé, qui se projette dans le futur, dans ses propres expériences. Par conséquent, j'ai préféré trois moments, qui présentent tous plus ou moins les mêmes thématiques, à une narration se déroulant d'un point à l'autre. De cette manière chaque thématique coexiste avec l'autre et elles agissent ensemble dans chaque mouvement.» (notre traduction, dorénavant T.d.A.), in Panzeri F. et Picone G., Tondelli. Il mestiere di scrittore, Ancône, Transeuropa, 1994, p. 74. 
aspects de la personnalité de Leo sont concernés: sa sexualité, la mise en question de sa stratégie amoureuse (celle des "chambres séparées»), ses origines, son idée de voyage entendu comme refuge et fuite de soi-même, son expérience religieuse de jeunesse, son rôle de père et de créateur qu'il ne vivra jamais, et qui est à l'origine du caractère sado-masochiste auquel toutes ses histoires d'amour aboutissent à un moment donné.

\section{La séduction comme expression du silence}

Le premier mouvement, qui s'intitule Verso il silenzio [Vers le silence], présente d'un côté la première rencontre entre Leo (dans lequel on reconnaît l'alter ego de l'auteur) et Thomas, et de l'autre la mort de Thomas. Le roman débute sur l'image de Leo dans un avion, en vol entre Paris et Munich. Il est plongé et presque englouti dans un état d' "abandon» (mot qui est, d'ailleurs, le titre d'un ouvrage de Tondelli ${ }^{3}$ ).

Dans les premières pages du roman - qui sont consacrées à la première rencontre entre les deux personnages - le champ sémantique le plus fréquent fait référence au sens de la vue. La séduction ne laisse pas de place à la parole; le seul langage possible est celui du corps, bien que les deux protagonistes appartiennent l'un au monde de la littérature (Leo) et l'autre à celui de la musique (Thomas).

Le lieu de la rencontre entre Leo et Thomas - la maison de Bernard est empli de lumière. Les objets, les personnes, les pièces de l'appartement, les corps se révèlent soudainement, comme dans un rêve, grâce à la lumière qu'ils diffusent vers l'extérieur: une lumière pénétrante, comme celle du regard de Leo se posant pour la première fois sur le corps de Thomas. Dans cette sorte de vision, Leo perçoit et découvre la «présence» de Thomas.

Le regard comme instrument de séduction n'est pas vraiment une nouveauté, surtout si l'on pense à la tradition littéraire du Moyen Age, aux premières expressions poétiques en vulgaire italien ${ }^{4}$. Mais dans le roman,

3. Tondelli P.V., L'abbandono. Racconti dagli anni ottanta, Milan, Bompiani, 1993 (posthume). Il s'agit d'un recueil d'écrits dans lesquels Tondelli réfléchit sur le rôle et la fonction de l'écriture et sur les différentes manières de travailler sur le langage avant de passer au véritable acte d'écriture. En particulier, l'auteur revient sur ses premiers modèles littéraires italiens (Arbasino, Celati) et sur la passion qu'il porte à la littérature américaine contemporaine.

4. Le regard représente dans la littérature médiévale le moyen, le véhicule et l'élément révélateur de l'amour et de la beauté. Il suffit de penser à la poésie provençale, à la poésie lyrique sicilienne de la cour de Frédéric II de Souabe, et surtout aux poètes du « dolce stil novo », entre autres, 
le regard n'a pas la fonction de contact ou de révélation de l'un à l'autre; il ne favorise pas non plus nécessairement le rapprochement de deux mondes, de deux identités. Au contraire, il marque comme une sorte de distance, de différence. Ce regard introspectif révèle plutôt l'anxiété d'un sentiment, d'un "connaître pour se re-connaître", d'un travail intense qui œuvre du dedans. Le regard cache et manifeste un sentiment de dérive et d'abandon: il devient le seuil et la frontière d'un jeu qui préfigure déjà une condition de séparation.

Le premier véritable contact entre Leo et Thomas a lieu lors d'un concert de rock ${ }^{5}$. L'excès de lumière et le mouvement agité, à la fois de Leo, pris dans sa recherche frénétique de Thomas, et de la foule, submergée de bruit et d'excitation, s'opposent à l'immobilité de Thomas, tranquillement assis parmi les spectateurs. Après un certain nombre de fatigantes acrobaties, Leo se fait remarquer de Thomas qui parvient enfin à le rejoindre ${ }^{6}$.

Dans ce passage du roman, nous retrouvons les caractéristiques principales de la séduction que l'un exerce sur l'autre. Au long des années passées avec Thomas, cette séduction provoquera chez Leo la recherche d'un état de "séparation" qui ensuite, après la mort de son compagnon, se transformera en solitude et en sentiment d'abandon. Dès ce premier contact, Thomas semble en effet s'adapter à l'étreinte de Leo, il s'y confond, il se serre contre lui, il se livre à Leo non pas en tant que Thomas, mais plutôt en tant que "Thomas-pour-Leo». Leo apparaît tout de suite comme celui qui choisit, domine, décide, qui unit et qui sépare : cette dynamique affective révèle la tendance sado-masochiste des sentiments de Leo, sur laquelle nous aurons l'occasion de revenir.

Le besoin, le transport et le contact physique que Leo ressent ne supposent jamais un sentiment d'union, ou la disponibilité à l'accueil dont Thomas fait au contraire toujours preuve à son égard. Si Thomas séduit Leo dans l'intention de trouver chez l'autre un «lieu » à habiter, Leo ne fait que se tourner de plus en plus vers lui-même, développant un «lieu de

Guido Guinizzelli et Dante. Dans la Vita Nova, par exemple, l'amour que Dante ressent pour Béatrice trouve son expression dans le regard qui est souvent discret et furtif et qui se substitue à la parole.

5. «Le luci sul palco lampeggiano intermittenti, colpi di decine e decine di flash rosa pastello [...] e finalmente il bianco accecante delle luci ad arco [...] un riflettore inizia a volteggiare fra il pubblico inquadrandolo in un ristretto e accecante cono di luce. [...] La luce sul palco è rosso intenso" (Camere separate, p. 24, 25).

6. "Leo lo guarda orgoglioso allargando le braccia. Thomas si adatta a quell'abbraccio, si confonde in esso, si stringe a Leo appoggiandogli la testa sulla spalla. Leo gli accarezza i capelli. [...] Le labbra di Leo cercano la bocca di Thomas» (p. 29). 
séparation » et de solitude. D'autre part, la relation avec Thomas place Leo face à l'obligation de désirer le contact avec une altérité que l'on puisse toucher, ressentir, que l'on vit et qui, par conséquent, le pousse à s'emparer de tout besoin idéal ${ }^{7}$.

Ce premier mouvement du roman s'achève sur la mort de Thomas, réduit à un état d'inconscience par sa maladie. Leo ne peut s'empêcher de poser encore une fois son regard sur les yeux souffrants de son compagnon. La séparation géographique, physique et existentielle imposée par Leo pendant la durée de leur relation se transforme maintenant, avec la mort de Thomas, en "séparation définitive». Aux yeux de Leo, Thomas devient soudainement un enfant. Son regard à demi éteint lui rappelle celui d'un enfant palestinien agonisant, d'un petit Indien d'Amérique du Sud qui assiste au massacre de sa race, tandis que Leo ressemble de plus en plus à un père impuissant qui sent son corps se vider de toute possibilité de rédemption avec soi-même et avec une histoire qui le fait définitivement entrer dans l'âge adulte.

\section{La séduction comme recherche de la solitude}

Le deuxième mouvement, intitulé Il mondo di Leo [Le monde de Leo], retrace les phases principales de la vie du personnage après la mort de Thomas, et revient sur les moments les plus importants de la relation entre les deux hommes. Tondelli nous fait part de leurs voyages à travers l'Europe, des efforts de Leo pour que les autres acceptent Thomas comme son «accompagnateur officiel» lors de ses conférences et de sa vie en société. En prenant progressivement conscience de l'attachement physique et psychique de Thomas, Leo sent grandir dans son cour un sentiment de peur ${ }^{8}$.

À côté de la narration en "absence» du personnage "Thomas-et-Leo", l'auteur continue de tracer le profil en "présence» du personnage «Leo-

7. «[Leo] sapeva, fin dall'inizio, che mai lui avrebbe potuto essere «tutto». Per questo chiamava il loro amore "camere separate». Lui viveva il contatto con Thomas come sapendo, intimamente, che prima o poi si sarebbero lasciati. La separazione era una forza costitutiva della loro relazione e ne faceva parte analogamente all'idea di attrazione, di crescita, di desiderio sessuale» (p. 101).

8. «Si voltò e vide, in quel fracasso, gli occhi di Thomas fissi nei suoi, solo per un istante, e allora sorrise, si alzò con tutti gli altri e uscirono dall'aula. In quel momento Leo sentì Thomas troppo profondamente accanto a sé. Sentì celebrata la sua unione, accettata, protetta, la sentì come un valore sociale fondamentale per difendere il quale un popolo avrebbe anche potuto affrontare una guerra, offrire una generazione intera al martirio pur di preservarlo intatto nel proprio patrimonio culturale» (p. 65-66). 
sans-Thomas». Dans cette contre-narration, l'univers de la vie - marqué par le passage de Leo de l'âge de l'adolescence à l'âge adulte - se reflète de façon presque spéculaire dans l'univers de la mort, représenté par le passage de Thomas de l'âge adulte à l'âge de l'enfance. Ce type d'écriture à deux visages est réalisé par une narration fragmentaire et discontinue, qui mêle l'histoire au souvenir, le passé au présent, tout en ouvrant une nouvelle perspective sur un "futur proche" où les effets de la mort semblent plus proches de Leo que de Thomas.

Dans cette sorte de contrepoint narratif spatio-temporel, Leo décide de partir pour un long voyage qui l'amènera en Angleterre, la "terre séparée " du continent européen. Il pourra enfin y éprouver sa condition d' «être séparé", tenter de fuir la mort de Thomas et de faire son travail de deuil dans une complète solitide ${ }^{9}$.

La séduction que Thomas avait exercée sur Leo à travers les sens, la vue, le corps, et qui avait engendré le personnage "Thomas-et-Leo", se transforme maintenant en un regard figé sur soi-même. Ce voyage de et vers la mort, tout en exprimant un évident refus de la vie, accompagne Leo jusqu'à sa complète destruction et, ensuite - après avoir "traversé» le corps mort de Thomas - à sa reconstruction finale. Cette conquête d'un nouvel équilibre passe chez Leo par une relecture radicale de son enfance, de sa passion pour l'écriture, de ses origines, de sa foi en un Dieu qui ne nie pas l'amour mais qui, au contraire, l'autorise dans toutes ses formes et ses expressions possibles. Réconforté par ce nouveau regard sur lui-même, Leo décide de se rendre à son village natal. Là aussi, il perçoit nettement un sentiment de séduction qui se transforme en mouvement, en un chemin vers l'essence de soi-même, vers un état de «séparation » qui n'est plus abandon de la vie, mais seulement abandon de la douleur.

Dans sa terre natale, souvenir de solitude et de non-acceptation, Leo parvient vraiment à relire sa vie: un parcours vers les origines, comme celui que Thomas avait effectué au moment de sa disparition. Leo découvre un village quitté depuis des années, mais cette fois il ne demande plus à faire partie d'un groupe, à être intégré au sein d'une communauté qui l'avait depuis toujours obligé à adhérer à des valeurs qui n'étaient pas dans sa nature. Pour la première fois son regard est chargé d'affection et de sou-

9. «Dopo circa un mese di piccoli spostamenti ora sta finalmente lasciando il continente e con esso il corpo martoriato di Thomas. Si lascia alle spalle la guerra, i cadaveri, il dolore, i campi di sterminio, le città distrutte e rase al suolo. L'Inghilterra gli appare come un paese separato e distante in cui non conosce nessuno e nessuno lo conosce, in cui può stare solo senza soffrire di solitudine, in cui può camminare, sedere al pub, bere, scrivere senza che nessuno lo guardi o lo disturbi. [...] Thomas era la Storia; il suo paese e la sua lingua gli scenari della guerra» (p. 69). 
venirs qui l'amènent à percevoir différemment les odeurs de sa jeunesse, les messes du dimanche, les vieilles dames qui bavardent sous les arcades. Ce lieu exerce une sorte de séduction irrépressible qui métamorphose son sentiment de "séparation" par rapport à cet univers familial. Chaque geste, chaque habitude, chaque rite familial devient le symbole d'une réalité transfigurée et lointaine. Leo comprend que même son amour pour l'écriture avait contribué à affirmer sa différence et à forger son imaginaire, son paysage idéal. La pratique de l'écriture avait mis en relief sa conscience de devenir de plus en plus un "être séparé»: cette même écriture qui, à présent, ne se manifeste plus, car Leo n'a plus de raisons de défendre sa différence ${ }^{10}$.

\section{La séduction comme désagrégation et reconstitution d'une identité}

Le désir de solitude qui aide Leo à construire son état de "séparation" reste la thématique centrale du troisième et dernier mouvement, qui s'intitule Camere separate [Chambres séparées]. Tout en revenant sur le motif inspirateur de son ouvrage, Tondelli insiste sur les derniers moments de l'existence du personnage "Thomas-et-Leo ». Il achève également le récit du voyage de Leo vers la conquête de son identité. Au niveau formel et narratologique, ce double plan de la narration crée une sorte de dyscrasie qui correspond à la genèse du principe des «chambres séparées ». Cette stratégie d'amour s'appuie sur l'idée d'un rapport fondé sur une relation de contiguïté, d'appartenance, mais jamais de possession.

Deux ans après leur première rencontre, Leo impose à Thomas une relation à distance faite de longues périodes d'absence, de silences, d'hésitations, de retrouvailles et d'abandons, qui deviennent pour Thomas une véritable prison de l'âme et du corps. Leo percevait une telle relation comme nécessaire, car elle convenait à sa manière sadomasochiste de vivre l'éros et la séduction de l'autre. Pour lui, la séduction devait conduire vers un ailleurs qui sépare, qui ne peut agir que dans un état d'absence, dans la crainte de devoir mourir et de disparaître. Le vide de ce rapport fondé sur

10. «Lui che aveva affidato alle parole, non ancora alla letteratura, non ancora ai libri, ma proprio alle lettere e ai racconti tutta l'ansia e il desiderio di un cambiamento della sua vita, si trova ora annullato dalla mancanza di desiderio per le parole. E conseguentemente, per le cose. [...] Lui è cosciente che il suo immaginario è morto. È cosciente di averlo perduto. E lo ha perso di fronte alla morte dell'amante, di fronte all'unica cosa che avrebbe potuto far crollare quella lunga, decennale, problematica, ambivalente calcificazione interiore di speranza e di sogno: la paura di dover morire, la paura di essere già morto" (p. 94, 141-142). 
la distance sera provisoirement comblé par l'écriture: Leo et Thomas décident en effet d'entamer une correspondance presque quotidienne pendant leurs périodes de séparation. L'action de la séduction quitte ainsi la sphère purement sensuelle et sexuelle pour agir à travers la parole. Le personnage "Thomas-et-Leo" essaie de s'ouvrir pour la première fois à l'idée d'altérité, et franchit la frontière de l'epos, d'une écriture qui tempère le vide de l'éloignement. L'écriture n'agit donc plus - comme pour Leo pendant sa jeunesse - en tant que dispositif qui affirme une différence et légitime le sentiment de non-possession de l'autre. Elle pousse, au contraire, à la rencontre de l'autre dans un ailleurs créé par le mouvement de séduction, de rencontre-collision, de présence-absence. Malgré l'effet bénéfique de l'écriture, Thomas, à la différence de Leo, n'arrive pas à s'habituer à l'absence et à la distance. Lors d'une de leurs rencontres à Berlin, Leo s'aperçoit enfin de l'illusion trompeuse de sa stratégie des "chambres séparées ${ }^{11}$.

Le détachement de Thomas bouleverse Leo, qui réalise que leur relation est probablement arrivée à un point de non-retour. Pendant les trois ans de leur relation, Leo n'avait cessé de tuer Thomas, dans le but de mystifier le sentiment de non-appartenance qu'il ressentait vis-à-vis de lui-même. Lorsque Leo pense avoir atteint le comble de son amour pour Thomas, la stratégie des "chambres séparées» prend une tournure imprévue. Thomas décide d'introduire dans leur espace et leur mouvement de séduction une troisième personne: une jeune fille prénommée Susann.

Se comportant comme un "anti-Leo", Susann arrive là où Leo ne veut pas ou ne peut pas arriver. À l'intérieur des «chambres séparées», Thomas est le seul à parcourir tous les espaces possibles. Il parvient ainsi à limiter la pulsion sadomasochiste de Leo et à se défendre de l'état de séparation qu'il lui impose. Si autrefois l'idée d'abandon jouait au détriment de Thomas, maintenant cette même idée fait prendre conscience à Leo qu'il a toujours désiré tout de Thomas et n'en a eu qu'une partie, un aspect, une projection.

Au sein du personnage "Thomas-et-Leo", les deux protagonistes ont progressé parallèlement, mais en sens inverse: tandis que Leo s'acheminait vers la prise de conscience - intime et silencieuse - de ses trente ans,

11. "Continuava a credere che l'amore fosse una meravigliosa vacanza della sua vita. Un weekend da passare insieme alla persona desiderata nella comodità, alla luce delle candele, con un paesaggio da ammirare e del buon rhum da bere. Forse "camere separate» era l'illusione, forse fin troppo turistica, che la sua idea dell'amore corrispondesse a quella delle canzoni e dei libri. Ma quale altro tipo di amore poteva esistere?» (p. 179). 
Thomas se dirigeait au contraire vers la récupération de sa jeunesse. Ce sentiment de non-appartenance sera aussi à la base de la dynamique qui s'instaure entre Thomas, Leo et Susann. L'équilibre de leur relation résulte de la synergie "en absence» de trois identités qui se mêlent sans jamais se posséder dans leur totalité.

Après la mort de Thomas, Leo poursuit son voyage en quête de solitude, vivant dans une sorte de chasteté forcée: il perçoit la disparition de Thomas comme une frontière, une manière de censurer et d'anesthésier ses sens et sa vie. Cette perte symbolise aussi la mort de Leo qui, dans son sentiment de déchirement, considère son corps comme quelque chose d'étranger et d'inutile, une carcasse infernale: un corps dont il renie les fonctions et les besoins qui le poussent parfois vers la perdition et la tentation sexuelle ${ }^{12}$.

La prise de conscience définitive de la mort de Thomas et le changement de vie de Leo qui en découle ont lieu grâce à l'intervention d'un ancien ami de Leo: Rodolfo. Il tente à plusieurs reprises de lui faire comprendre qu'il faudrait célébrer une fois pour toutes les funérailles de Thomas, de sorte que sa vie puisse enfin être réellement vécue. Dès lors, la recherche de la solitude de la part de Leo ne se limite plus à un pur mouvement intérieur et autoréférentiel. Au contraire, la douleur que Leo a ressentie jusqu’à présent parvient à faire naître une forme de vie: le désir de se recomposer et de former une famille. Dans son prototype de famille, il n'y a de place ni pour une femme ni pour des enfants, ni pour des pères ou des mères. En revanche, il y a de la place pour des individus qui fondent entre eux un lien aussi fort et aussi naturel. Parmi les membres de cette famille on retrouve Rodolfo, Michael et Eugenio. Ce dernier est un jeune garçon que Leo apprendra à connaître et à apprécier après quelques difficultés initiales (et surtout grâce à la considération qu'Eugenio manifeste pour son activité littéraire).

Après des années vécues dans la vaine recherche d'une prison capable de garder intacte la mémoire de Thomas, le regard de Leo se porte vers le "dehors»: pour qu'il puisse atteindre non pas la paix illusoire d'une quiétude initiale ou d'un passé révolu, mais une nouvelle perception des choses et de l'homme, de l'autre et de soi-même ${ }^{13}$.

12. "Ma il fatto più grave è che la sua rinuncia gli sta rendendo il corpo sempre più inconoscibile e estraneo. La castità è una virtù mistica, per quanti l'hanno scelta; e forse l'uso sovrumano della sessualità. Per lui questa astinenza coatta è solamente una tortura in più che sta trasformando in ossessione il suo naturale bisogno di corporalità, di contatti, di carezze, di visione del corpo nudo dell'altro" (p. 191-192).

13. "[Leo] deve finalmente procedere al disimpasto fra chi è vivo e chi è morto. Annullare l'avvoltoio che è in lui. Perché se una cosa è certa è che per tutti questi anni lui si è mantenuto in 
Lorsque Leo rencontre Eugenio - l'étymologie grecque de ce prénom renvoie à l'idée de "bonne naissance» -, il apprend à vivre la séduction différemment: elle n'est plus simplement un sentiment qui lie à une autre personne, mais une sensation physique et intellectuelle qui permet de ressentir tout ce qui caractérise l'espèce humaine. Ainsi, le personnage "Thomas-et-Leo " ne représente pas une entité stérile, comme Leo le pensait du vivant de Thomas. Ce personnage uni et séparé, proche et lointain, en présence et en absence, a su engendrer quelque chose, quelqu'un: le "Leo-adulte", qui arrive à sortir de son monde pour toucher, comprendre, sentir et aimer un monde qui ne s'arrête plus au corps mort de Thomas ${ }^{14}$. Le regard de séduction physique, qui avait eu une place remarquable au moment de la rencontre entre les deux hommes, se transforme donc en regard intériorisé qui mène à la maturité et à l'achèvement d'un projet humain, se traduisant en témoignage et en une prise de conscience de l'identité individuelle ${ }^{15}$.

Ce parcours vers la lumière - qui jaillit et éblouit avec force, sémantiquement et symboliquement, dans un certain nombre de pages de ce roman - concerne aussi la réflexion de Leo sur son activité littéraire et, par conséquent, le questionnement de Tondelli par rapport au rôle et à la fonction de la littérature, dans une époque que l'on qualifie de "postmoderne». L'écriture n'est plus le lieu esthétique d'une fuite, d'un idéal, d'une identité violée par un narcissisme aux nuances sadomasochistes. Elle est au contraire un lieu de veille et de séduction, de rencontre et d'ouverture, de douleur et de compréhension: un espace ouvert, où tout peut se déplacer dans une sorte de jeu d'agrégation et de désagrégation de la matière, des sentiments et des sensations.

Le roman se termine sur le voyage de Leo à Québec, à l'occasion d'une rencontre organisée pour célébrer la mémoire de Jack Kerouac. Pendant son séjour, Leo fait un bilan "provisoirement» définitif de son travail de deuil, et se rend compte que son destin d' "homme séparé» sera lié pour toujours à celui de "Leo-écrivain». Cet état de «séparation» ne se fonde

vita cibandosi delle spoglie mortali di Thomas, dissetandosi con il suo sangue, sfamandosi con la sua carne squarciata come un qualsiasi insaziabile predatore di carcasse della savana» (p. 196).

14. «Un tempo, quando riteneva la sua relazione d'amore sterile e senza frutto, si sbagliava. Leo e Thomas hanno partorito, con dolore, almeno un figlio. E questo figlio espulso nel mondo, che pensa e agisce, è oggi il trentatreenne Leo» (p. 203).

15. Fulvio Panzeri, dans sa "Nota al testo", écrit ceci : "Dans Chambres séparées Tondelli célèbre une sorte de rite, celui du retour, accompagné d'une pietas soumise: un retour qui définit les raisons de la prise de conscience de soi dans une solitude qui donne lieu aux élégies d'une fragmentation s'imposant sur l'existence à partir du niveau littéraire» (T.d.A.). In Tondelli P.V., Biglietti agli amici, Milan, Bompiani, 1997, p. 109. 
plus sur la conviction d'être un étranger qui met en scène sa vie, mais sur celle d'être un témoin actif de la séduction. Il s'agit d'une séduction entendue comme création de quelque chose qui n'existe pas encore, mais qui demande d'ores et déjà d'être aimée et qui se construit dans un corps à corps avec la vie ${ }^{16}$.

\section{Pour une parabole de la séduction}

La parabole du personnage de Leo est marquée par une séduction qui illustre toute une phénoménologie complexe de l'abandon (dans le sens, à la fois, d'un rapprochement et d'une séparation). Thomas se livre sans condition à Leo, acceptant la confiance qu'une séduction silencieuse et sensuelle installe rapidement entre eux. Au contraire, Leo vit le sentiment d'abandon comme un éloignement de Thomas, du fait d'un manque de conscience de soi et d'un sentiment de non-appartenance à lui-même. Cet abandon devient enfin définitif lors de la mort de Thomas. Sa disparition est d'ailleurs à l'origine du voyage de Leo dans son intériorité.

Cette double nature du sentiment d'abandon se reflète dans les trois étapes fondamentales que le personnage "Thomas-et-Leo» traverse: l'élaboration de la stratégie des "chambres séparées", qui marque la fuite de Leo loin d'une passion destructrice; la déconstruction de son identité, qui se réalise avec la mort de Thomas, avec la disparition du personnage "Thomas-et-Leo", et avec la réflexion sur le sens et le rôle qu'a pris l'écriture dans sa vie; enfin, la réhabilitation de Leo qui retrouve le sens de la vie et de l'écriture en fonction de l'ouverture à l'autre.

La séduction agit fortement dans la vie de Leo. Au bout de son voyage, il prend conscience de l'urgence de sortir de la "représentation", de la «mise en scène» de son existence, pour accomplir sa recherche de l'essentiel et faire ainsi de la solitude un moyen d'apporter des réponses à ses besoins et à ses désirs. Cela veut dire être prêt à récuser un idéal entendu comme prison et différence, pour s'ouvrir à une solitude entendue comme valeur, dans le but d'accéder à une passion qui soit surtout compréhension de l'Homme. La condition de «l'être séparé» n'est plus seulement une

16. «La sua diversità, quello che lo distingue dagli amici del paese in cui è nato, non è tanto il fatto di non avere un lavoro, né una casa, né un compagno, né figli, ma proprio il suo scrivere, il dire continuamente in termini di scrittura quello che gli altri sono ben contenti di tacere. La sua sessualità, la sua sentimentalità si giocano non con altre persone, come lui ha sempre creduto, finendo ogni volta con il rompersi la testa, ma proprio nell'elaborazione costante, nel corpo a corpo, con un testo che ancora non c'è» (p. 212). 
manière d'affirmer une différence, mais surtout une attitude qui amène l'homme au sacrificium et qui prépare l'accueil de l'Autre.

Au long de ce parcours, la séduction - dans le sens de «se porter ailleurs» - passe par différents niveaux de manifestation. À la séduction sensuelle, celle du corps et du regard, succède une séduction "dans l'absence» à travers la mémoire, l'écriture et la parole. Elle s'insinue dans l'intimité de l'individu, afin qu'il puisse se retrouver là où personne ne l'attend, dans un corps à corps avec une vie et une écriture qui sont encore à concevoir et à créer. La séduction est dès lors le terrain où les notions de possession et de non-appartenance se rejoignent enfin sous le signe commun du désir et de la connaissance. 